\title{
A sharp bound for the reconstruction of partitions
}

\author{
Vincent Vatter \\ Department of Mathematics \\ Dartmouth College \\ Hanover, NH 03755
}

Submitted: May 16, 2008; Accepted: Jun 22, 2008; Published: Jun 30, 2008

Mathematics Subject Classification: 05A17, 06A07

\begin{abstract}
Answering a question of Cameron, Pretzel and Siemons proved that every integer partition of $n \geq 2(k+3)(k+1)$ can be reconstructed from its set of $k$-deletions. We describe a new reconstruction algorithm that lowers this bound to $n \geq k^{2}+2 k$ and present examples showing that this bound is best possible.
\end{abstract}

Analogues and variations of Ulam's notorious graph reconstruction conjecture have been studied for a variety of combinatorial objects, for instance words (see Schützenberger and Simon [2, Theorem 6.2.16]), permutations (see Raykova [4] and Smith [5]), and compositions (see Vatter [6]), to name a few.

In answer to Cameron's query [1] about the partition context, Pretzel and Siemons [3] proved that every partition of $n \geq 2(k+3)(k+1)$ can be reconstructed from its set of $k$-deletions. Herein we describe a new reconstruction algorithm that lowers this bound, establishing the following result, which Negative Example 2 shows is best possible.

Theorem 1. Every partition of $n \geq k^{2}+2 k$ can be reconstructed from its set of $k$-deletions.

We begin with notation. Recall that a partition of $n, \lambda=\left(\lambda_{1}, \ldots, \lambda_{\ell}\right)$, is a finite sequence of nonincreasing integers whose sum, which we denote $|\lambda|$, is $n$. The Ferrers diagram of $\lambda$, which we often identify with $\lambda$, consists of $\ell$ left-justified rows where row $i$ contains $\lambda_{i}$ cells. An inner corner in this diagram is a cell whose removal leaves the diagram of a partition, and we refer to all other cells as interior cells.

We write $\mu \leq \lambda$ if $\mu_{i} \leq \lambda_{i}$ for all $i$; another way of stating this is that $\mu \leq \lambda$ if and only if $\mu$ is contained in $\lambda$ (here identifying partitions with their diagrams). If $\mu \leq \lambda$, we write $\lambda / \mu$ to denote the set of cells which lie in $\lambda$ but not in $\mu$. We say that the partition $\mu$ is a $k$-deletion of the partition of $\lambda$ if $\mu \leq \lambda$ and $|\lambda / \mu|=k$.

Recall that this order defines a lattice on the set of all finite partitions, known as Young's lattice, and so every pair of partitions has a unique join (or least upper bound)

$$
\mu \vee \lambda=\left(\max \left\{\mu_{1}, \lambda_{1}\right\}, \max \left\{\mu_{2}, \lambda_{2}\right\}, \ldots\right)
$$


and meet

$$
\mu \wedge \lambda=\left(\min \left\{\mu_{1}, \lambda_{1}\right\}, \min \left\{\mu_{2}, \lambda_{2}\right\}, \ldots\right) .
$$

Finally, recall that the conjugate of a partition $\lambda$ is the partition $\lambda^{\prime}$ obtained by flipping the diagram of $\lambda$ across the NW-SE axis; it follows that $\lambda_{i}^{\prime}$ counts the number of entries of $\lambda$ which are at least $i$.

Before proving Theorem 1 we show that it is best possible:

Negative Example 2. For $k \geq 1$, consider the two partitions

$$
\begin{aligned}
\mu & =(\underbrace{k+1, \ldots, k+1}_{k}, k-1) \text { and } \\
\lambda & =(\underbrace{k+1, \ldots, k+1}_{k-1}, k, k) .
\end{aligned}
$$

Note that no $k$-deletion of $\mu$ can contain the cell $(k, k+1)$ and that no $k$-deletion of $\lambda$ can contain the cell $(k+1, k)$. Therefore every $k$-deletion of $\mu$ and of $\lambda$ is actually a $(k-1)$-deletion of

$$
\mu \wedge \lambda=(\underbrace{k+1, \ldots, k+1}_{k-1}, k, k-1),
$$

so $\mu$ and $\lambda$ cannot be differentiated by their sets of $k$-deletions.

We are now ready to prove our main result.

Proof of Theorem 1. Suppose that we are given a positive integer $k$ and a set $\Delta$ of $k$ deletions of some (unknown) partition $\lambda$ of $n \geq k^{2}+2 k$. Our goal is to determine $\lambda$ from this information. We begin by setting $\mu=\bigvee_{\delta \in \Delta} \delta$, noting that we must have $\lambda \geq \mu$. Hence if $|\mu|=n$ then we have $\lambda=\mu$ and we are immediately done, so we will assume that $|\mu|<n$.

First consider the case where $\mu$ has less than $k$ rows. Let $r$ denote the bottommost row of $\mu$ which contains at least $k$ cells ( $r$ must exist because $\mu$ has less than $k$ rows and $\left.|\mu| \geq k^{2}+k\right)$. Thus the $r$ th row of $\lambda$ contains at least $k$ cells as well, so there are $k$-deletions of $\lambda$ in which the removed cells all lie in or below row $r$. Hence the first $r-1$ rows of $\lambda$ and $\mu$ agree. Now note that $\lambda$ has more than $2 k$ cells to the right of column $k$, so there are $k$-deletions of $\lambda$ in which the removed cells all lie to the right of column $k$, and thus the first $k$ columns of $\lambda$ and $\mu$ agree. This implies that $\lambda$ and $\mu$ agree on all rows below $r$ (since these rows have less than $k$ cells in $\mu$ ) and so all cells of $\lambda / \mu$ must lie in row $r$, uniquely determining $\lambda$, as desired. The case where $\mu$ has less than $k$ columns follows by symmetry.

We may now assume that $\mu$ has at least $k$ rows and $k$ columns. Let $r$ (resp. $c$ ) denote the bottommost row (resp. rightmost column) containing at least $k$ cells. Both $r$ and $c$ exist because $\mu$ has at least $k$ rows and columns. Therefore both $\lambda$ and $\mu$ can be divided into three quadrants, 1, 2, and 3, as shown in Figure 1.

As before, we see that the first $r-1$ rows and $c-1$ columns of $\lambda$ and $\mu$ agree. We consider three cases based on whether and where $r$ and $c$ intersect. 


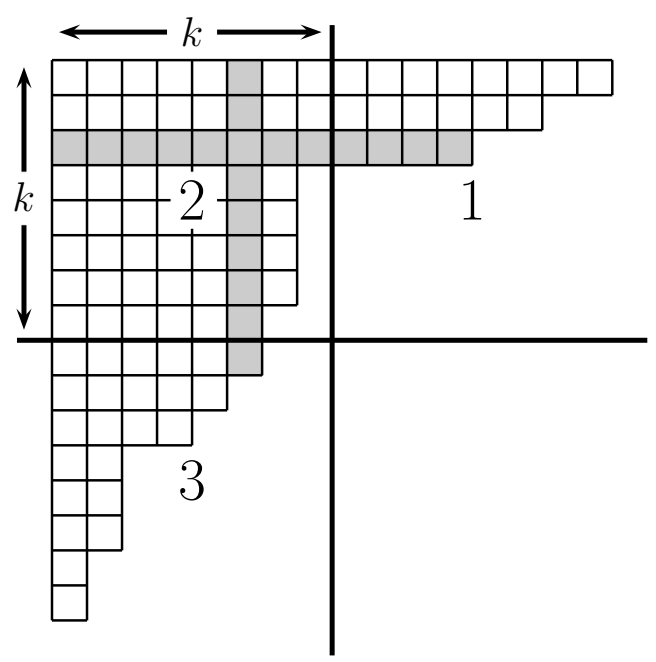

Figure 1: An example partition $\mu$ from Case 1 of the proof of Theorem 1, divided into three quadrants. Here $k=8$, and $r$ and $c$ appear shaded.

Case 1: $r$ and $c$ intersect at an interior cell of $\mu$. Suppose that $r$ and $c$ intersect at the cell $(i, j)$. It follows from the maximality of $r$ and $c$ that $i, j<k$, and thus the cell $(k, k)$ does not lie in $\mu$. Were the cell $(k, k)$ to lie in $\lambda$ then, because $|\lambda| \geq k^{2}+2 k, \lambda$ must contain at least $2 k$ cells to the right of or below $(k, k)$ and thus $\lambda$ would contain a $k$-deletion with the cell $(k, k)$, a contradiction; thus $\lambda$ also does not contain $(k, k)$.

Hence Quadrant 2 of $\lambda$ contains less than $k^{2}$ cells, so $\lambda$ must have more than $k$ cells in quadrant 1 or 3 . Hence there are $k$-deletions of $\lambda$ with more than $k$ cells in quadrant 1 or 3 ; suppose by symmetry that $\lambda$ and $\mu$ both have more than $k$ cells in quadrant 1 .

There are then $k$-deletions of $\lambda$ in which the removed cells are all chosen from quadrant 1 , so $\lambda$ and $\mu$ agree on all cells in quadrants 2 and 3 . This shows that $r$ is also the bottommost row of $\lambda$ with at least $k$ cells, and so $\lambda / \mu$ contains no cells below row $r$ in quadrant 1. As we already know that $\lambda$ and $\mu$ agree on their first $r-1$ rows, we can therefore conclude that all cells of $\lambda / \mu$ lie in row $r$, which allows us to reconstruct $\lambda$ and complete the proof of this case.

Case 2: $r$ and $c$ intersect at an inner corner of $\mu$. Then this inner corner must be the rightmost cell of row $r$ and the bottom cell of column $c$. It follows that $r, c \geq k$. Because $\lambda$ and $\mu$ agree to the left of column $c$ and above row $r$, all cells of $\lambda / \mu$ must lie below or to the right of $(r, c)$. However, the cell $(r+1, c+1)$ cannot lie in $\lambda$ because if it did then one could form a $k$-deletion of $\lambda$ by removing only points lying to the right of column $c$, which would leave at least $k$ cells in row $r+1$ and contradict the definition of $r$. This leaves only two possibilities for $\lambda / \mu$ : the cells $(r, c+1)$ and $(r+1, c)$. However, only one of these cells can be added to $\mu$ to produce a partition; if both could be added then row $r+1$ and column $c+1$ of $\lambda$ would each contain at least $k$ cells, implying the existence of $k$-deletions of $\lambda$ in which each contain at least $k$ cells and thus contradicting the choice of $r$ and $c$. This case therefore reduces to checking which one of the cells $(r, c+1)$ and $(r+1, c)$ can be added to $\mu$ to produce a partition. 
Case 3: $r$ and $c$ do not intersect. Suppose that the rightmost cell in row $r$ is $(r, j)$ and the bottommost cell in column $c$ is $(i, c)$. If $j<c-1$ then because $\lambda$ and $\mu$ agree to the left of column $c, \lambda / \mu$ cannot contain any cells in or below row $r$, and we already have that $\lambda$ and $\mu$ agree above row $r$, so we are left with the conclusion that $\lambda=\mu$. By symmetry we are also done if $i<r-1$, leaving us to consider the case where $i=r-1$ and $j=c-1$. Again using the fact that $\lambda$ and $\mu$ agree above row $r$ and to the left of column $c$ (and the definitions of $r$ and $c$ ) we see that the only possibility for $\lambda / \mu$ is $(r, c)$, completing the proof of this case and the theorem.

Acknowledgements. I would like to thank the referee for several suggestions which improved the transparency of the proof.

\section{References}

[1] Cameron, P. J. Stories from the age of reconstruction. Congr. Numer. 113 (1996), $31-41$.

[2] Lothaire, M. Combinatorics on Words, vol. 17 of Encyclopedia of Mathematics and its Applications. Addison-Wesley Publishing Co., Reading, Mass., 1983.

[3] Pretzel, O., and Siemons, J. Reconstruction of partitions. Electron. J. Combin. 11, 2 (2004-06), Note 5, 6 pp.

[4] Raykova, M. Permutation reconstruction from minors. Electron. J. Combin. 13 (2006), Research paper 66, 14 pp.

[5] Smith, R. Permutation reconstruction. Electron. J. Combin. 13 (2006), Note 11, 8 pp.

[6] Vatter, V. Reconstructing compositions. Discrete Math. 308, 9 (2008), 1524-1530. 